\title{
Multiple-Field Approach for Aberration Correction in Miniature Imaging Systems Based on Wafer-Level Production
}

\author{
Eric Logean ${ }^{* a}$, Toralf Scharf ${ }^{a}$, Nicolas Bongard $^{a}$, Hans Peter Herzig ${ }^{a}$, Markus Rossi $^{b}$ \\ ${ }^{a}$ Optics \& Photonics Technology Laboratory, Ecole Polytechnique Fédérale de Lausanne \\ (EPFL), Rue A.-L. Breguet 2, 2000 Neuchâtel, Switzerland; \\ ${ }^{b}$ Heptagon, 8803 Rüschlikon, Switzerland
}

\begin{abstract}
In mobile imaging systems, the most difficult element to integrate is the objective lens. Here we present an intermediate approach between the costly traditional objectives and the low-resolution objectives inspired by the compound eyes of insects. Our multi-field approach uses a small number of optical channels each imaging a portion of the desired field of view. The full-field image is reconstructed digitally. The optics of each channel is kept simple for wafer-level fabrication and its size is sufficient to obtain a reasonable resolution. We present the design and fabrication of a prototype using 9 plano-convex lenses for 9 channels. Glass lenses glued on a wafer are used to image a full-field of $\pm 40^{\circ}$ with an $f$-number of 3 . The images obtained shows field curvature correction. A simple image reconstruction scheme is presented. In conclusion, multi-field objectives fabricated with micro-optics technology are thin, simple to mount, robust, and easily replicated.
\end{abstract}

Keywords: Imaging systems, micro-optics, geometric optical design, aberration compensation, optical design and fabrication, image reconstruction techniques.

\section{INTRODUCTION}

The camera objective is the most difficult element to integrate into nomadic devices. In addition to the significant thickness of the objective, its mounting requirements are often difficult to meet and are costly. These problems stem from the traditional approach to aberration control using a series of lenses placed sequentially along the optical axis. ${ }^{1}$ Novel approaches include the use of the wavefront coding method to simplify the lens systems ${ }^{2,3}$ and multi-channels objectives. ${ }^{4-9}$ Of the multi-channel objectives, the work of Brückner et al. is attractive. ${ }^{10}$ These authors fabricated a thin array of numerous small channels each imaging a field of $10^{\circ}$. The full-field image covers $58^{\circ} \times 46^{\circ}$. The information from overlapping fields are used to digitally increase the reconstructed image angular resolution using a technique similar to Tanida et al. ${ }^{5}$

In our work, we concentrate on intermediate optical systems between the traditional objective and the multifaced eye. An analogy could be drawn with the visual system of the jumping spiders (Portia) and their eight eyes. ${ }^{11}$ Our designs have a small number of separated channels to simplify the channel separation. The size of the channel aperture is adequate to produce images with an angular resolution close to the resolution of the human eye. The optics is required to be simple for fabrication using wafer-level techniques, ${ }^{12-14}$ resulting in optical elements ready for mass production.

Here we present the design of our multi-field objective using single plano-convex lenses working at an $f$-number of 3 and covering a full-field of $\pm 40^{\circ}$. We describe the fabrication method used at our facilities. Then, the objectives are built and characterised. Simple image acquisition and reconstruction are presented. The results are discussed and placed in perspective with what can be done in state of the art factories.

* eric.logean@epfl.ch

Multimedia Content and Mobile Devices, edited by David Akopian, et al., Proc. of SPIE-IS\&T Electronic Imaging, SPIE Vol. 8667, 86671E · @ 2013 SPIE-IS\&T · CCC code: 0277-786X/13/\$18 · doi: 10.1117/12.2004390 


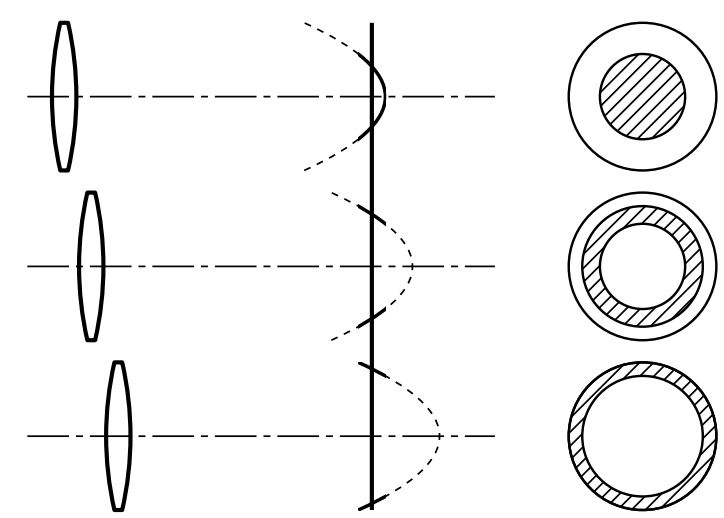

Figure 1. Drawing of three lens systems forming three images on a flat sensor represented by a vertical continuous line (centre). The three lenses are identical. They form sharp images over a curved field (dashed line). Each lens is located at a different axial position and its curved field crosses the sensor at a different height. On the right, the three fields are represented. The hatched areas indicate the field areas sharply imaged on the sensor.

\section{MULTI-FIELD IMAGING}

The advantage of a multi-field imaging system over a conventional objective is an added degree of freedom. Each channel can perform a different task. Concepts such as the foveated system ${ }^{15,16}$ and the split-field ${ }^{17}$ can be implemented. For aberration control, the task of each channel is limited to a given sub-field. This situation is advantageous because the amount of most optical aberrations varies rapidly over different field areas. ${ }^{18}$

The image produced by a single-lens system lies on a curved surface. This aberration is called field curvature and it can not be reduced in such a simple system. The effect of a curved field could be avoided by using a curved image sensor. ${ }^{19,20}$ Unfortunately, these sensors are not yet commercially available and are not compatible with wafer-level low-cost production techniques. Within the multi-field framework, field curvature can be corrected by changing the optical power of each channel. This approach was demonstrated - as well as astigmatism correction - using carefully designed elliptical micro-lenses of varying radii of curvature. ${ }^{21}$ As it is technically challenging to produce array of lenses of different power, we propose to use lenses of identical power located at different distances from the sensor as depicted in Fig. 1. On the left side, three lens systems are drawn. These systems form three separated images on a flat image sensor represented by the vertical line. Each lens is located at a different axial position and its curved field crosses the sensor at different heights. The part of the field which appears sharp on the sensor is represented by a continuous line. On the right, the three fields are sketched. For each system, the hatched area represents the sharply imaged part of the field.

\subsection{Plano-convex objective}

A plano-convex lens has a small number of free parameters. For a given material, the desired lens power sets the radius of curvature of the convex surface. The remaining free parameters are the lens thickness and the aperture stop size and position. By locating the aperture stop at the plane surface of the lens and by setting the thickness of the lens equal to the radius of curvature of the convex surface, as shown in Fig. 2, the lens is free from coma, astigmatism, and lateral chromatic aberration. ${ }^{18}$ For this stop position, the amount of both distortion and field curvature is large. The image distortion can be corrected digitally, ${ }^{23}$ but the effect of field curvature has to be corrected optically. Here we use the multi-field concept as shown in Fig. 1. The remaining first order aberrations are the spherical aberration and the longitudinal chromatic aberration. For the lens dimension considered, spherical aberration has a stronger effect than longitudinal chromatic aberration. It can be reduced using an aspheric lens profile or by reducing the aperture of the stop.

\subsection{Optical design}

Due to our current limitation, we are unable to fabricate lenses with the required diameter. Therefore, we rely on commercially available spherical lenses (45223, Edmund Optics, USA) having a diameter of $1.0 \mathrm{~mm}$ and a focal length of $1.0 \mathrm{~mm}$. The optical design is tailored to a colour webcam sensor (C600, Logitech, Switzerland) 


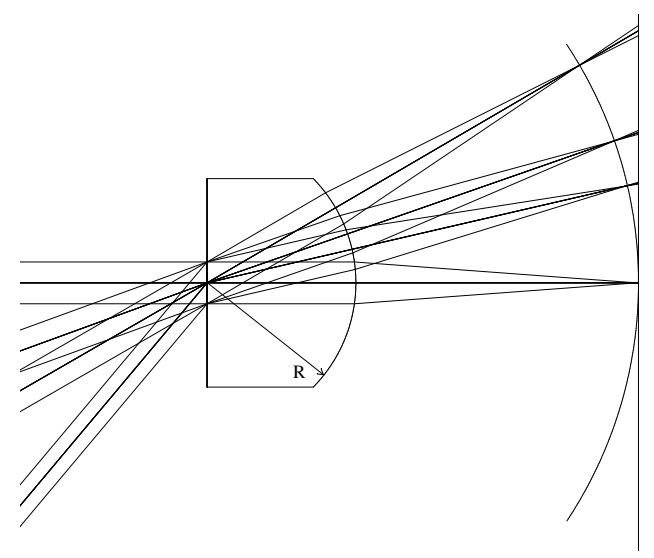

Figure 2. Schematic drawing of the thick plano-convex lens with stop at the plane surface, a design close to the rear landscape lens ${ }^{22}$ but with a single curved surface. The arrow indicates that the centre of curvature of the convex surface is at the centre of the aperture.

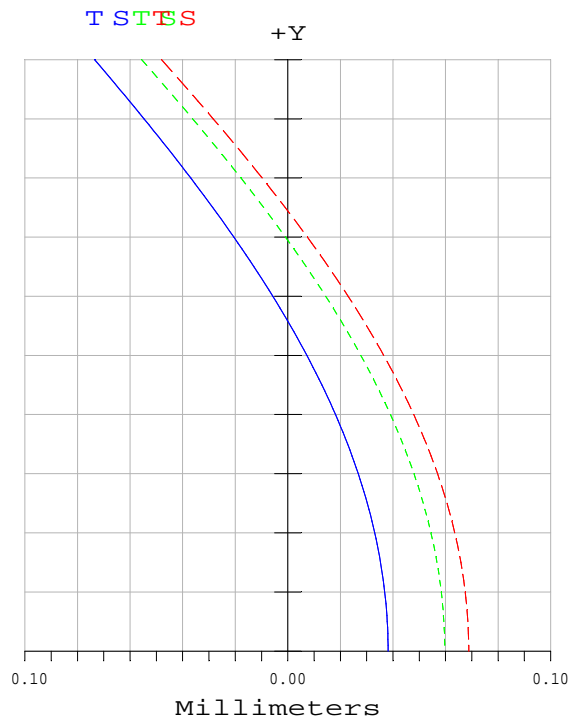

Figure 3. Plot of the field curvature for the designed lens (diameter of the aperture stop of $0.33 \mathrm{~mm}$ ) for field angles up to $40^{\circ}$ and for the wavelength of $486 \mathrm{~nm}$ (blue, continuous), $589 \mathrm{~nm}$ (green, short dash), $656 \mathrm{~nm}$ (red, long dash).

having $1600 \times 1200$ square pixels with a side length of $2.835 \mu \mathrm{m}$. The sensing area is $4.536 \mathrm{~mm} \times 3.416 \mathrm{~mm}$. It is covered by a Bayer filter and a glass window. We set the acceptable blur diameter $w=10 \mu \mathrm{m}$, a value close to the length of 4 pixels. Then ray tracing software or geometrical optics calculation ${ }^{18,24}$ can be used to determine the maximum radius of the stop that limits the image blur due to spherical aberration to the acceptable blur $w$. We found a radius $a$ of $0.166 \mathrm{~mm}$ giving an $f$-number of 3 .

The depth of focus of this lens system is $60 \mu \mathrm{m}$. Using a ray tracing software (Radiant Zemax, USA), we generated the plots of field curvature shown in Fig. 3 for a field angle up to $40^{\circ}$ and for three wavelengths: 486, 589 , and $656 \mathrm{~nm}$. The axial displacement between the blue (continuous) and the red (long dash) curves reveals a maximum longitudinal chromatic aberration of $30 \mu \mathrm{m}$ on axis, a value much smaller than the depth of focus. Therefore, the effect of longitudinal chromatic aberration on the image quality is expected to be small. However, the maximum focus shift which is due to field curvature is $115 \mu \mathrm{m}$, a value twice as large as the approximated depth of focus. This aberration seriously degrades the image quality. Using two lens systems (channels) axially shifted by $60 \mu \mathrm{m}$, a sharp image of the total field can be acquired. 


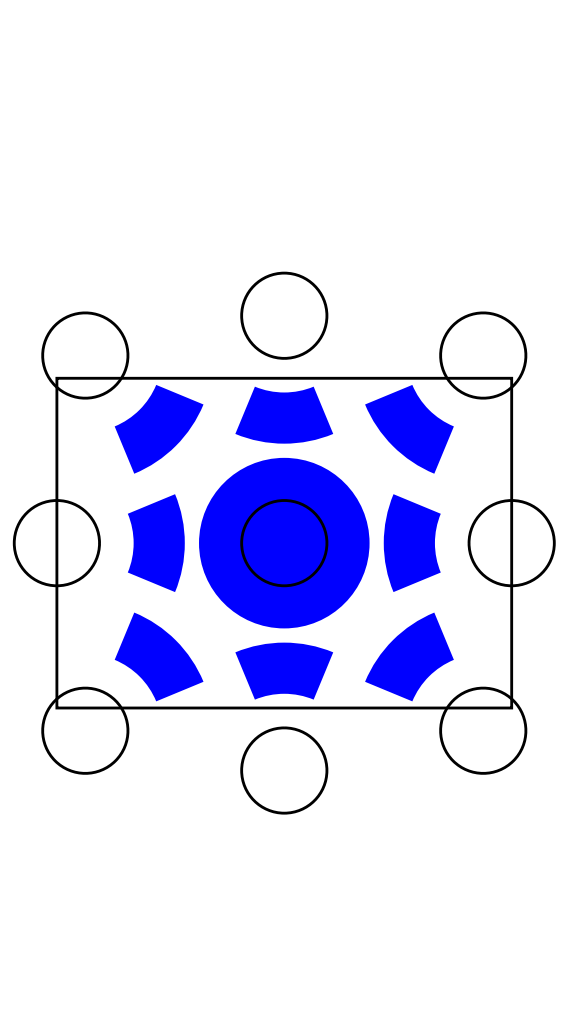

(a)

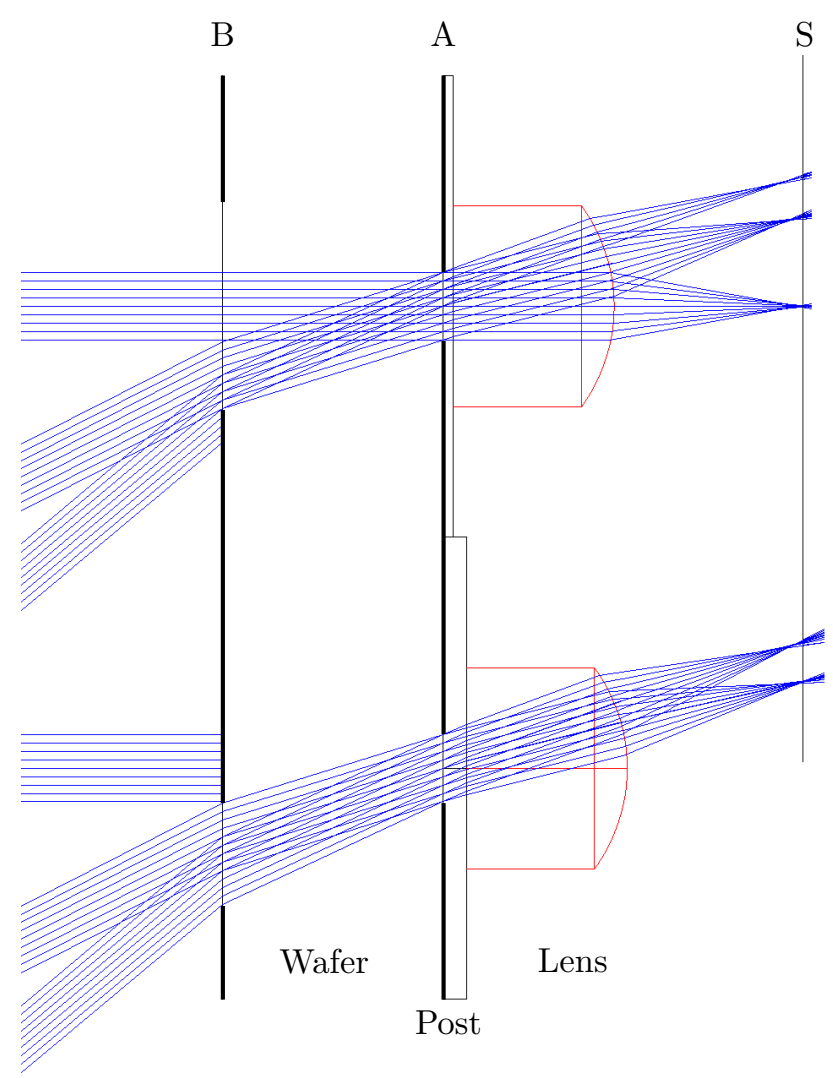

(b)

Figure 4. (a) Schematic drawing of the field layout, the circles represent the position of the aperture stops, the rectangle represents the sensor area, and the blue zones represent the field image from each channels. (b) Cross-section drawing of the designed optical system passing through the centre lens (top) and the satellite lenses (bottom). The $1.1 \mathrm{~mm}$ thick wafer support posts of different thickness onto which the lenses (in red) are glued. The front surface of the wafer (B) is covered with black photoresist with aperture used to separate the different fields. The back surface (A) is also covered with black photoresist with aperture defining the channel stops. The sensing surface is on the right (S). Rays from three different field angle: $0^{\circ}, 27^{\circ}$, and $40^{\circ}$ are traced for both channels (blue).

\subsection{System layout and field curvature correction}

The optical systems we constructed consisted of a central plano-convex lens that image a field of $\pm 27^{\circ}$ and satellite channels that extend the field to $40^{\circ}$. For a good use of the sensor area, the field extension is divided into eight annular sectors of $45^{\circ}$ and arranged as depicted in Fig. 4 (a). In that drawing, the circles represent the position of the channel apertures, the rectangle represents the sensor area, and the blue areas represent the useful part of the image from each channels.

A cross-section view of the central channel and of one of the satellite channels is shown in Fig. 4 (b). Apertures are formed on both sides of a glass wafer. On the back side (A), we have the aperture stops. On the front side (B), limiting apertures are used to avoid light cross-talk between the different channels. The lenses are glued on the back side. The satellite lenses are on a post used to compensate the field curvature. On the right, the image sensor $(\mathrm{S})$ is represented. The designed total track length is $3.0 \mathrm{~mm}$.

\section{FABRICATION}

The designed multi-field objectives are fabricated using circular glass wafers with a diameter of 4 inches and with a thickness of $1.1 \mathrm{~mm}$ (D263T, Schott, Germany). On a single wafer, 16 squares modules with a side length of $11 \mathrm{~mm}$ are fabricated in parallel. The two sides of the wafer were covered by black photoresist (EK410, 


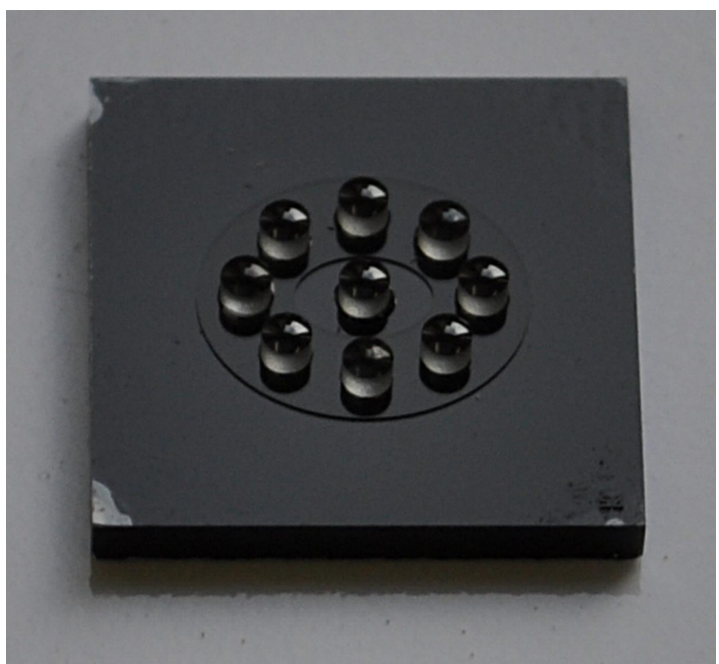

(a)

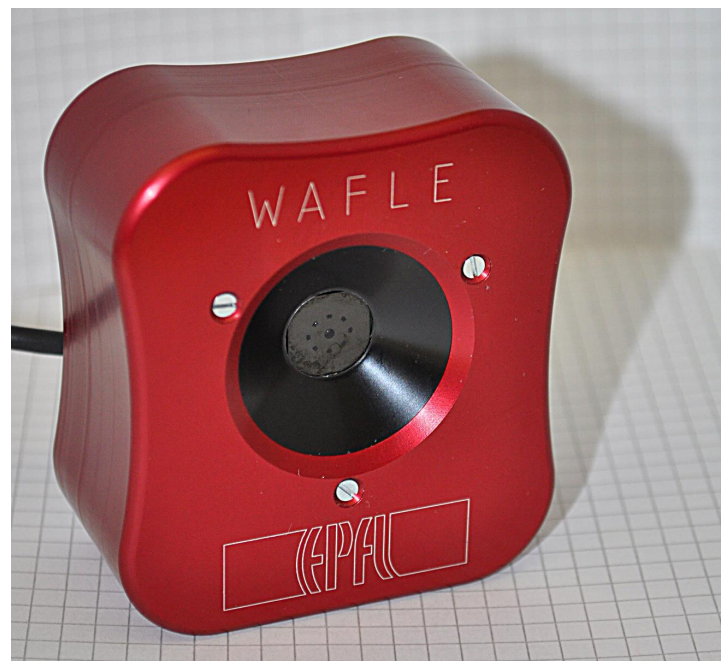

(b)

Figure 5. (a) Image of the back-side of the fabricated objective. It is square with a side length of $11 \mathrm{~mm}$. (b) The finished device. The objective is glued onto the black support and aligned in front of the camera sensor. The dimensions of the case are $6.0 \times 5.5 \times 3 \mathrm{~mm}^{3}$.

Everlight Chemical Industrial Co., Taiwan). Photolithographic steps are used to form apertures on both sides. On the back side of the wafer, the aperture stops with a diameter of $0.33 \mathrm{~mm}$ are formed. On the front side, limiting apertures are formed to avoid light cross-talk between the different channels. A $60-\mu m$ thick layer of glue (NOA83H, Norland Products Inc., USA) was replicated and polymerised on the back side of the wafer. Finally, the 9 glass lenses are aligned using two micro-manipulators, under a microscope, and they are glued. A photograph of the multi-field objective is shown in Fig. 5 (a). We measured the lens centring relative to each aperture stop and we found an average shift of $13 \mu \mathrm{m}$. The multi-field objective is then glued on a circular support, it is aligned in front of the camera and enclosed into a dedicated box as seen in Fig. 5 (b).

\section{EXPERIMENTAL RESULTS}

Figure 6 shows an image of a square grid super-imposed with marks delimiting the field to be imaged by each channel. The inner-circle extends to a field angle of $27^{\circ}$ and the outer-circle to $40^{\circ}$. The central image is sharp up to a field angle of $27^{\circ}$. For larger field angle, the image sharpness and contrast decrease. Each satellite channel images its dedicated field sharply and without vignetting.

The effect of the field curvature correction is directly visible in Fig. 6. However, to quantify the improved image quality, we measured the modulation transfer function (MTF) at the three locations shown in Fig. 6: (1) in the centre of the image of the central channel, (2) at a field angle of $33^{\circ}$ on the image of the central channel (at $6 \mathrm{~h}$ ), and (3) at a field angle of $33^{\circ}$ on the image of the corresponding satellite channel (top). We used the slanted edge method ${ }^{25,26}$ to avoid aliasing due to the low sampling resolution of the camera. The MTF-curve for the three image locations are shown in Fig. 7. The MTF curve for the field angle of $33^{\circ}$ of the central channel image (2) is lower than the two other curves. It reaches a modulation of 0.3 for a spatial frequency of $60 \mathrm{cycles} / \mathrm{mm}$. For the two other curves, that is for the central location (1) and for the corrected satellite image (3), a modulation better than 0.3 is obtained up to a spatial frequency of 90 cycles $/ \mathrm{mm}$. These results match the MTF obtained from ray tracing (not shown). The standard deviation obtained from 10 repeated measurements is smaller than 0.05 .

For image reconstruction, the four corners of the annular sectors were used as landmarks in a linear, twodimensional mapping routine using Matlabß (The Mathworks, Inc., USA). Figure 8 (a) shows the raw image of the grid from the central channel as in Fig. 6. Figure 8 (b) shows the digitally reconstructed image. The mapped area in the reconstructed image is clearly visible due to uncorrected brightness and colour variations from the 


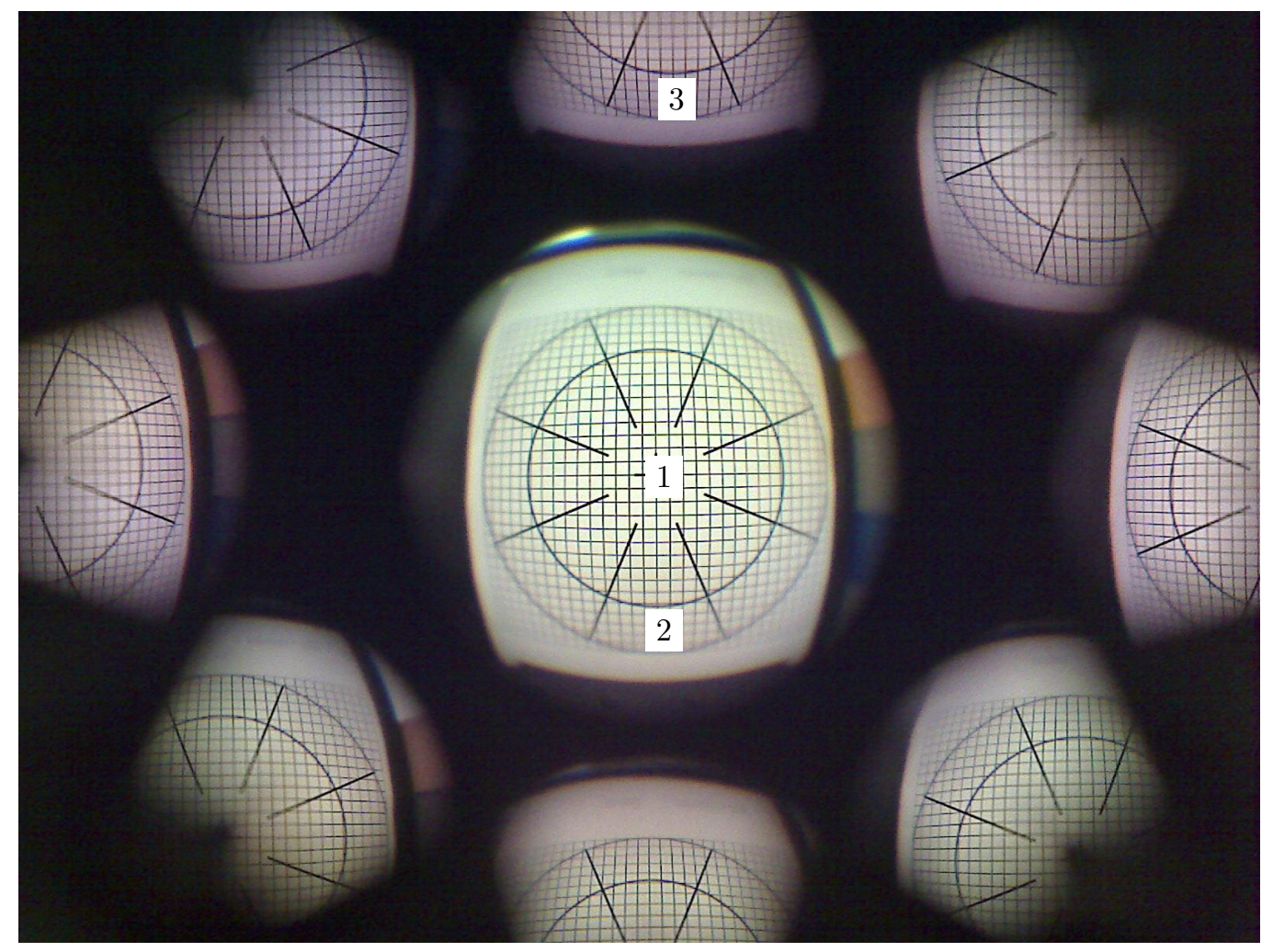

Figure 6. Raw image of a grid acquired with the multi-field objective. The central circle extends to a field angle of $27^{\circ}$ and the external one to $40^{\circ}$. The central image is sharp up to about $27^{\circ}$. The satellite images are sharp between 27 to $40^{\circ}$ as designed. The number 1 to 3 indicate the location of the modulation transfer function measurements shown in Fig. 7.

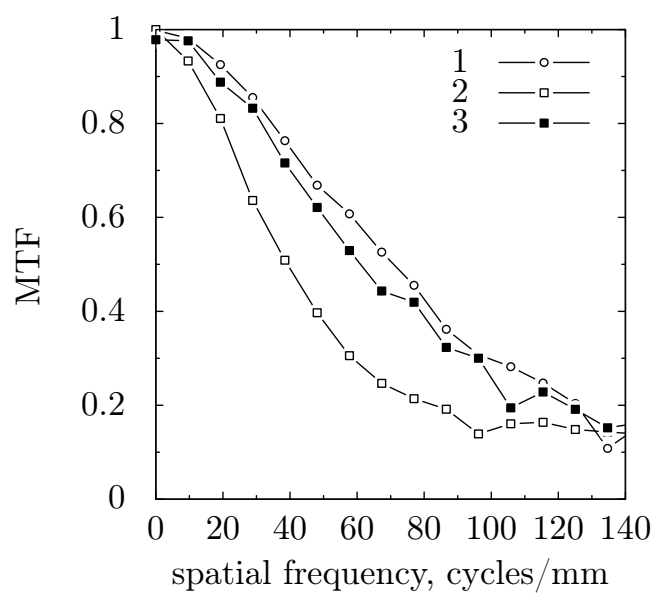

Figure 7. Measured MTF plot for the three image locations shown in Fig. 6. The location (1) is in the centre and (2) at a field angle of $33^{\circ}$ of the central channel image. The location (3) is in the satellite image corresponding to the location (2). 


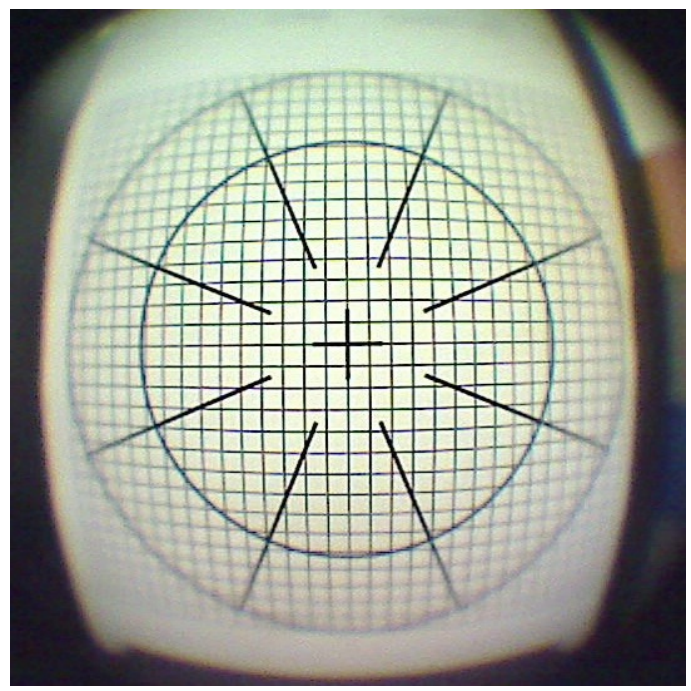

(a)

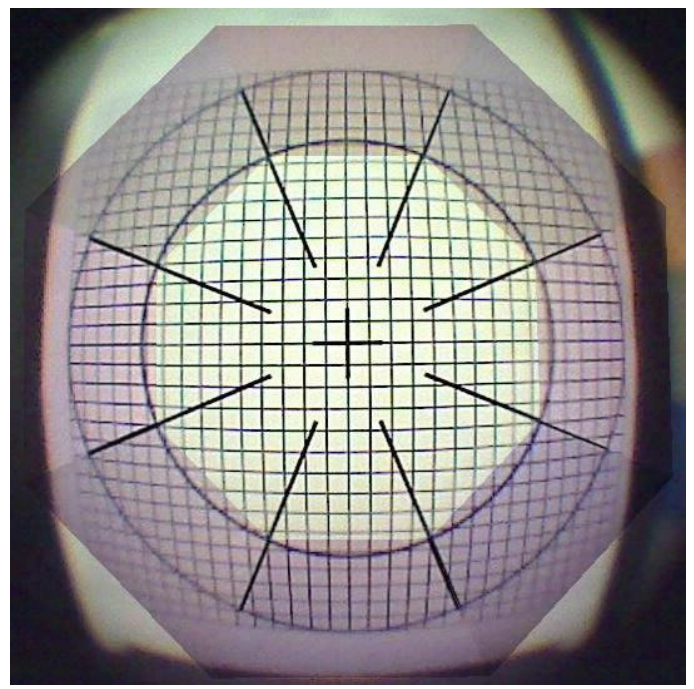

(b)

Figure 8. (a) Raw image of the central channel from Fig. 6, (b) Reconstructed image obtained by mapping the images of the satellite channels.

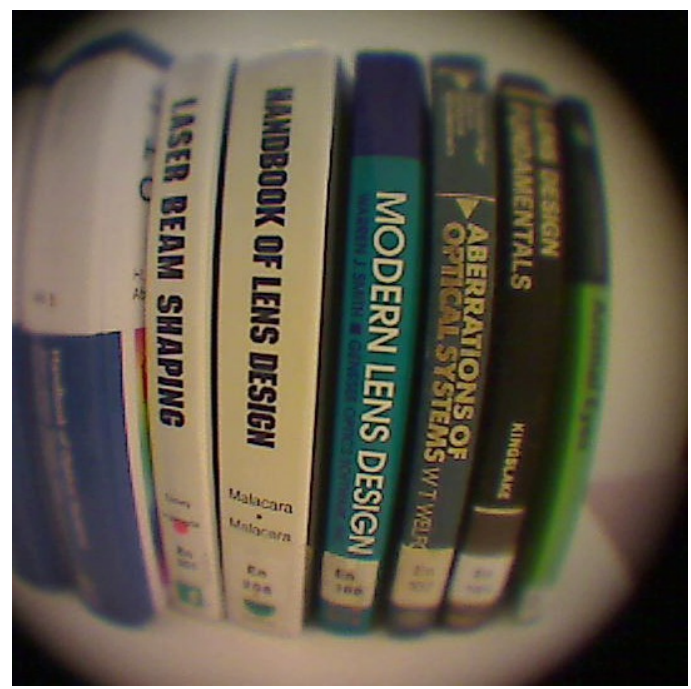

(a)

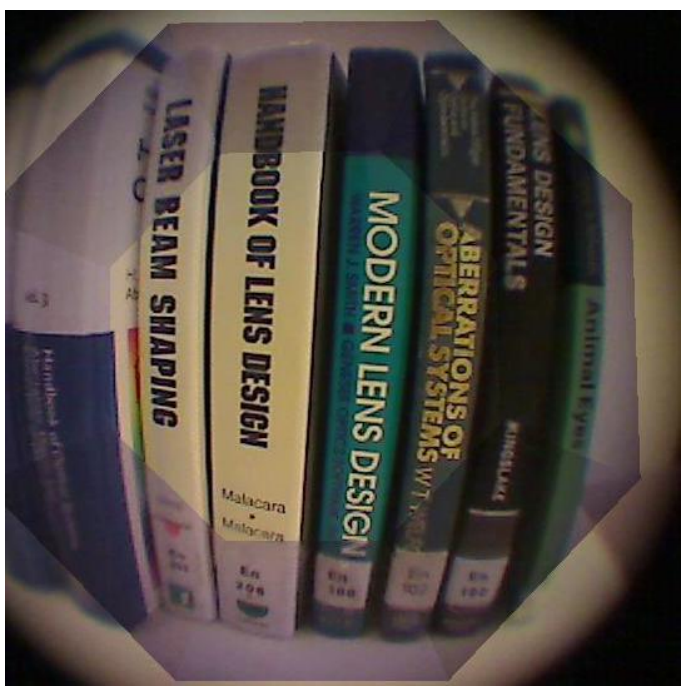

(b)

Figure 9. (a) Raw image of the central channel showing a few books, (b) Reconstructed image obtained by mapping the images of the satellite channels.

raw image. These effects, as well as the strong barrel distortion, can be corrected digitally. In this close-up view, the improved image sharpness in each $45^{\circ}$-sectors is clearly visible.

The reconstruction mapping obtained using the grid image is now used to reconstruct the images acquired with the multi-field objective. Figure 9 shows the image of a few books. The writings on the mapped area are easier to read in the corrected image than in the original image. Figure 10 shows the image of an origami crane. The crane wing-span is $12 \mathrm{~cm}$. To acquire this image, the camera was placed at about 40 to $50 \mathrm{~mm}$ to the crane. At that small distance, a small parallax effect is visible at the junction between the crane head and its neck.

\section{CONCLUSION}

We have designed and fabricated a multi-field imaging system using plano-convex lenses on a single wafer. The system track length is $3.0 \mathrm{~mm}$. It is small and simple to align in front of an image sensor. The strong field 


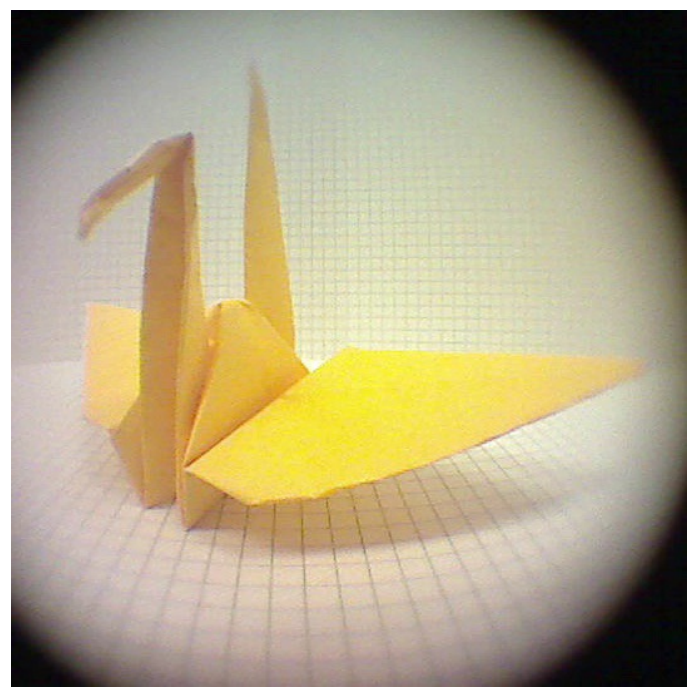

(a)

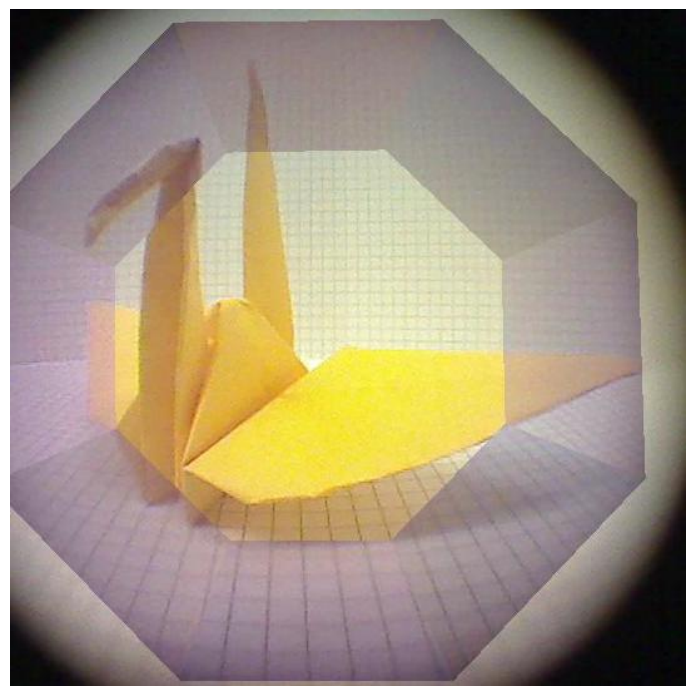

(b)

Figure 10. (a) Raw image of an origami crane, (b) Reconstructed image obtained by mapping the images of the satellite channels.

curvature inherent to single positive lens system is corrected using a number of separated channels for different part of the field of view. The system ability to correct field curvature was measured. Reconstructed images were obtained using simple image mapping.

Digital processing of the images is required for the multi-field concept. The full-field image has to be assembled using the sub-field images from all channels. In addition, the image distortion, colour, and brightness have to be corrected. All these operations can be integrated into the embedded electronic of current image sensors. Although, a manual intervention was required to glue the lenses, our multi-field objective can be replicated using moulding technology. Furthermore, in a state-of-the-art facilities, lenses of the required dimension can be fabricated at the wafer level using the reflow technique ${ }^{12,13}$ enabling, cheap production and the possibility to correct spherical aberration using an aspheric lens profile.

The objective presented here, is an example of a multi-field objective. By adding a second wafer between the lenses and the sensor, inter-channel cross talk can be strongly reduced and a better usage of the sensing area can be obtained. The total track lens can also be reduced. The objective is also very different from the one presented by Brückner et al. ${ }^{10}$ It illustrates the variety of design that can be obtained when the design emphasises any of the following parameters: Number of channels, channel separation and cross-talk suppression, image brightness, information redundancy, track length. Furthermore, the added design freedom obtained using the multi-field concept may be used to implement different techniques, ${ }^{15-17}$ to vary the optical properties of each channel for user-defined functions, and possibly the obtain depth information using channel parallax.

\section{ACKNOWLEDGMENTS}

We thanks Irène Philipoussis Fernandez for her technical expertise, Durgesh Kumar for his help with the image reconstruction step, and Mitsuko Logean for the origami crane. This work was supported by the Eurostars project 4303 WAFLE.

\section{REFERENCES}

[1] Smith, W. J., [Modern Lens Design: A Resource Manual], McGraw-Hill, New York, ${ }^{\text {st }}$ ed. (1992).

[2] Dowski, Jr., E. R. and Cathey, W. T., "Extended depth of field through wave-front coding," Appl. Opt. 34(11), 1859-1866 (1995).

[3] Cathey, W. T. and Dowski, E. R., "New paradigm for imaging systems," Appl. Opt. 41(29), 6080-6092 (2002). 
[4] Hamanaka, K. and Koshi, H., "An artificial compound eye using a microlens array and its application to scale-invariant processing," Opt. Rev. 3(4), 264-268 (1996).

[5] Tanida, J., Kumagai, T., Yamada, K., Miyatake, S., Ishida, K., Morimoto, T., Kondou, N., Miyazaki, D., and Ichioka, Y., "Thin observation module by bound optics (TOMBO): Concept and experimental verification," Appl. Opt. 40(11), 1806-1813 (2001).

[6] Jeong, K.-H., Kim, J., and Lee, L. P., "Biologically inspired artificial compound eye," Science 312(5773), $557-561(2006)$.

[7] Druart, G., Guérineau, N., Haïdar, R., Thétas, S., Taboury, J., Rommeluère, S., Primot, J., and Fendler, M., "Demonstration of an infrared microcamera inspired by Xenos Peckii vision," Appl. Opt. 48(18), 3368-3374 (2009).

[8] Brückner, A., Duparré, J., Wippermann, F., Dannberg, P., and Bräuer, A., "Microoptical artificial compound eyes," in [Flying Insects and Robots], Floreano, D., Zufferey, J.-C., Srinivasan, M. V., and Ellington, C., eds., ch. 10, 127-142, Springer-Verlag, Heidelberg (2009).

[9] Li, L. and Yi, A. Y., "Development of a 3D artificial compound eye," Opt. Express 18(17), 18125-18137 (2010).

[10] Brückner, A., Duparré, J., Leitel, R., Dannberg, P., Bräuer, A., and Tünnermann, A., "Thin wafer-level camera lenses inspired by insect compound eyes," Opt. Express 18(24), 24379-24394 (2010).

[11] Land, M. F. and Nilsson, D.-E., [Animal Eyes], Oxford Animal Biology Series, Oxford University Press, Oxford, UK (2002).

[12] Völkel, R., Eisner, M., and Weible, K. J., "Miniaturized imaging system," Microelectron. Eng. 67-68, 461-472 (2003).

[13] Rossi, M., Rudmann, H., Marty, B., and Maciossek, A., "Wafer-scale micro-optics replication technology," in [Proc. SPIE: Lithographic and Micromachining Techniques for Optical Component Fabrication II], Kley, E.-B. and Herzig, H. P., eds., 5183, 148-154 (2003).

[14] Herzig, H. P., ed., [Micro-Optics. Elements, Systems and Applications], Taylor \& Francis, London, UK (1997).

[15] Martinez, T., Wick, D. V., and Restaino, S. R., "Foveated, wide field-of-view imaging system using a liquid crystal spatial light modulator," Opt. Express 8(10), 555-560 (2001).

[16] Wick, D. V., Martinez, T., Restaino, S. R., and Stone, B. R., "Foveated imaging demonstration," Opt. Express 10(1), 60-65 (2002).

[17] Nayar, S. K., "Computational cameras: Redefining the image," Computer 39(8), 30-38 (2006).

[18] Kidger, M. J., [Fundamental Optical Design], vol. PM92, SPIE Press, Bellingham, Washington, USA (2002).

[19] Ko, H. C., Stoykovich, M. P., Song, J., Malyarchuk, V., Choi, W. M., Yu, C.-J., III, J. B. G., Xiao, J., Wang, S., Huang, Y., and Rogers, J. A., "A hemispheral electronic eye camera based on compressible silicon optoelectronics," Nature 454(7205), 748-753 (2008).

[20] Rim, S.-B., Catrysse, P. B., Dinyari, R., Huang, K., and Peumans, P., "The optical advantages of curved focal plane arrays," Opt. Express 16(7), 4965-4971 (2008).

[21] Duparré, J., Wippermann, F., Dannberg, P., and Reimann, A., "Chirped arrays of refractive ellipsoidal microlenses for aberration correction under oblique incidence," Opt. Express 13(26), 10539-10551 (2005).

[22] Kingslake, R. and Johnson, R. B., [Lens Design Fundamentals], SPIE Press, Oxford, $2^{\text {nd }}$ ed. (2010).

[23] Ricolfe-Viala, C. and Sanchez-Salmeron, A.-J., "Lens distortion models evaluation," Appl. Opt. 49(30), $5914-5928$ (2010).

[24] Gross, H., Zügge, H., Peschka, M., and Blechinger, F., [Handbook of Optical Systems], vol. 3: Aberration Theory and Correction of Optical Systems, Wiley, Darmstadt (2007).

[25] Burns, P. D., "Slanted-edge MTF for digital camera and scanner analysis," in [Proc. ISET 2000 PICS Conference], 135-138 (2000).

[26] Burns, P. D. and Williams, D., "Refined slanted-edge measurements for practical camera and scanner testing," in [Proc. ISEST 2002 PICS Conference], 191-195 (2002). 